\title{
Observation of Saddle-Point Avoidance in Noise-Induced Escape
}

\author{
D. G. Luchinsky, ${ }^{1,2}$ R. S. Maier, ${ }^{3,4}$ R. Mannella, ${ }^{5}$ P. V. E. McClintock, ${ }^{1}$ and D. L. Stein ${ }^{4,3}$ \\ ${ }^{1}$ Department of Physics, Lancaster University, Lancaster LA1 4YB, United Kingdom \\ ${ }^{2}$ Russian Research Institute for Metrological Service, Ozernaya 46, 119361 Moscow, Russia \\ ${ }^{3}$ Department of Mathematics, University of Arizona, Tucson, Arizona 85721 \\ ${ }^{4}$ Department of Physics, University of Arizona, Tucson, Arizona 85721 \\ ${ }^{5}$ Dipartimento di Fisica, Università di Pisa and INFM UdR Pisa, Piazza Torricelli 2, 56100 Pisa, Italy
}

(Received 6 October 1998)

\begin{abstract}
The first measurements of an exit location distribution are reported for an overdamped nonconservative system perturbed by weak white noise, modeled both numerically and by an analog electronic circuit. In the weak-noise limit the distribution is increasingly concentrated near a saddle point of the dynamics and is increasingly well approximated by a Weibull distribution, in agreement with theoretical predictions. A physical explanation for this behavior is given, which should facilitate the computation of corrections to the limiting form. [S0031-9007(99)08581-6]
\end{abstract}

PACS numbers: 05.40.Ca, 02.50.-r, 05.20.-y, 07.50.Ek

A fundamental problem in the fluctuational dynamics of randomly perturbed multistable systems is that of computing an exit location distribution, i.e., the probability distribution of exit points on the boundary $\partial \Omega$ of the domain of attraction $\Omega$ of a stable state. This problem appears in many areas of science and technology, e.g., in statistical physics [1,2], chemical physics [3-5], fluid mechanics [6], ecology $[7,8]$, and telecommunications $[9,10]$.

In the familiar case of a noise-perturbed overdamped system in equilibrium, exit from the domain of attraction $\Omega$ preferentially takes place in the vicinity of a saddle point of the deterministic (zero-noise) dynamics. In the two-dimensional case, the exit location distribution on $\partial \Omega$ typically converges in the limit of weak noise to a Gaussian centered on the saddle point, whose width decreases with ticular interest to the chemical physics community [3-5], but are usually due to a comparatively large noise intensity and/or anisotropic diffusion.

More recently, attention has focused on the problem of noise-induced exit in an overdamped, nonequilibrium system (e.g., one whose noise-free dynamics are nonconservative, so that even in stationarity there is nonzero probability current [11]). The boundary of the domain of attraction of a stable point may then contain no saddle points. More typically, the boundary contains one or more saddle points, and noise-induced exit tends to occur preferentially in the vicinity of one of them. But the phenomenology of escape differs considerably from the equilibrium case $[12,13]$. In general, the exit location distribution on $\partial \Omega$ is now skewed: it no longer resembles a Gaussian, even in the limit of weak noise [9,10,14-18].

In two-dimensional nonequilibrium systems, the skewing behavior is largely determined by $\mu=\left|\lambda_{s}\right| / \lambda_{u}$, the ratio of the stable and unstable eigenvalues of the linearized deterministic dynamics at the saddle point [14-18]. The $\mu<1$ case is particularly striking in that the exit location distribution in the $\epsilon \rightarrow 0$ (weak-noise) limit, besides being noise intensity. Deviations from this behavior are of par-

skewed, is localized on a much broader length scale near the saddle point than the $O\left(\epsilon^{1 / 2}\right)$ length scale that dimensional analysis would predict. Maier and Stein [15] have shown that if $\mu<1, O\left(\epsilon^{\mu / 2}\right)$ is the correct length scale. They have also predicted that as $\epsilon \rightarrow 0$, the exit location distribution on $\partial \Omega$ typically converges on this length scale to a Weibull, or "stretched exponential" distribution. It is a one-sided distribution: In the weak-noise limit, the system state in most noise-perturbed nonequilibrium models tends to avoid the saddle point, by exiting from $\Omega$ a small distance to one side of it. This is a more fundamental form of "saddle-point avoidance" than the type seen in conservative systems [5].

Direct comparison with experiment has become desirable. In this Letter we use analog and digital experiments to seek the predicted phenomenon of skewing, and we relate it to the geometry of the most probable escape path (MPEP) from the stable state to $\partial \Omega$, as it approaches the saddle point. The anomalously broad exit location distributions when $\mu<1$ are predicted to have a simple physical origin: the MPEP grazes $\partial \Omega$ as it approaches the saddle point [15]. (See, e.g., Fig. 1.)

The system we investigate is a bistable one: an overdamped particle moving in the symmetric, twodimensional drift field

$$
\mathbf{u}(x, y)=\left[x-x^{3}-\alpha x y^{2},-\mu y\left(1+x^{2}\right)\right],
$$

where $\alpha$ and $\mu$ are parameters [16]. This system has stable points at $( \pm 1,0)$, a separatrix or "ridge" (i.e., $\partial \Omega$ ) along the $y$ axis, and a saddle point at $(0,0)$. If isotropic white noise $\mathbf{f}(t)=\left(f_{x}, f_{y}\right)$ of strength $\epsilon$ is added, the equations of motion of the particle become

$$
\begin{aligned}
\dot{x} & =x-x^{3}-\alpha x y^{2}+f_{x}(t), \\
\dot{y} & =-\mu y\left(1+x^{2}\right)+f_{y}(t), \\
\left\langle f_{i}(t)\right\rangle & =0, \quad\left\langle f_{i}(s) f_{j}(t)\right\rangle=\epsilon \delta_{i j} \delta(s-t) .
\end{aligned}
$$




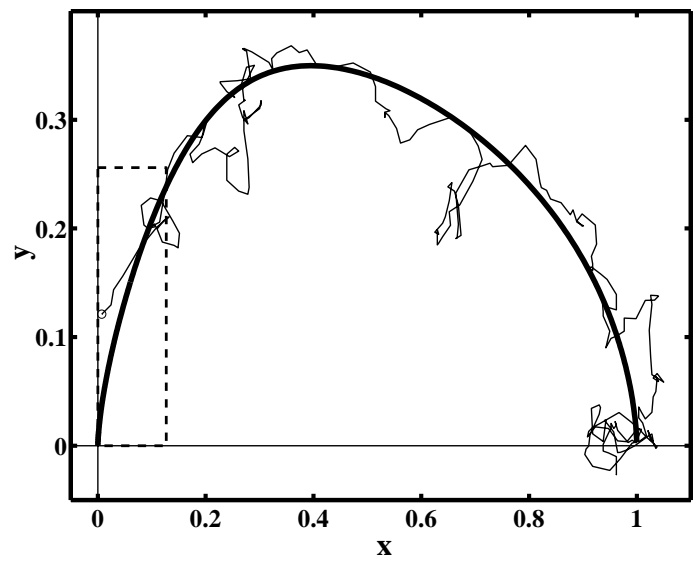

FIG. 1. First-quadrant MPEP (thick, solid line) for $\mu=0.66$ and $\alpha=10$, compared to a typical escape path (thin, jagged line) seen using analog simulation. The dashed rectangle represents the length scale near $(0,0)$ on which the exit location distribution is localized for $\epsilon=0.016$. Thin straight lines show separatrix $x=0$ and the other axis of symmetry $y=0$.

In the language of chemical physics, $x$ is a reaction coordinate, $y$ is a nonreacting coordinate, and the half-planes $x>0$ and $x<0$ (in either order) correspond to the reactant and product wells. Since $\dot{x} \approx x$ and $\dot{y} \approx-\mu y$ near $(0,0),\left|\lambda_{s}\right| / \lambda_{u}$ equals $\mu$.

Because of the symmetry through the $x$ axis, for many choices of parameters there are two MPEPs extending from each stable point to the saddle point $(0,0)$ [19]. Each grazes the $y$ axis, and together they yield a limiting "symmetrized Weibull" distribution on the $y$ axis near $y=0$. So, one-sidedness of the exit location distribution should here be replaced by bimodality.

To test these predictions, we have built an analog electronic model [20] of (2). We drive it with zero-mean quasiwhite Gaussian noises from a noise generator, digitize the response $x(t), y(t)$, and analyze it with a digital data processor. We have also carried out a complementary digital simulation, using a high-speed pseudorandom generator [21] for the increments $(\Delta x, \Delta y)$ because simulation times necessarily grow exponentially as $\epsilon \rightarrow 0$. In both analog and digital simulations, trajectories from the stable state $(+1,0)$ to first crossings of the $y$ axis were measured, and the corresponding exit location distributions were built and analyzed.

Our theoretical prediction for the exit location distribution was based on the following analysis. The phenomenon of noise-induced exit in the $\epsilon \rightarrow 0$ limit is governed by the slowest decaying eigenmode $\rho_{1}(x, y)$ (quasistationary density) of the Fokker-Planck operator $\mathcal{L}_{\epsilon}=$ $(\epsilon / 2) \nabla^{2}-\mathbf{u} \cdot \nabla-\nabla \cdot \mathbf{u}$ corresponding to (2). The absorption location density of this mode on the $y$ axis determines the exit location density $p_{\epsilon}(y)$. With increasing accuracy as $\epsilon \rightarrow 0$,

$$
p_{\epsilon}(y) \propto \partial_{x} \rho_{1}(0, y) .
$$

Our prediction for the exit location distribution relies on an approximation to $\rho_{1}$ that is increasingly valid as $\epsilon \rightarrow 0$. The approximation also yields predictions for the limiting MPEPs. The appropriate tool for constructing this approximation is the method of matched asymptotic approximations [7,22]. Away from the stable state and the $y$ axis, $\rho_{1}(x, y)$ should take on, to leading order, a WKB form const $\times \exp [-W(x, y) / \epsilon]$. Near the saddle point $(0,0)$, this form should match an approximation of the "turning point" type.

The eikonal function $W(x, y)$ of the WKB approximation (also called a "quasipotential" [12]) has a classicalmechanical interpretation. For any point $(x, y)$ in the domain of attraction of either stable state, $W(x, y)$ is the action of the least-action zero-energy trajectory of an auxiliary Hamiltonian system that extends from the stable state to $(x, y)$. The Hamiltonian function $H(\mathbf{x}, \mathbf{p})$ for the auxiliary system is $\frac{1}{2} \mathbf{p}^{2}+\mathbf{p} \cdot \mathbf{u}(\mathbf{x})$.

These zero-energy trajectories satisfy Hamilton's equations, and are the most probable fluctuational trajectories of the noise-perturbed system. Such "optimal" trajectories are experimentally observable, and the momentum $\mathbf{p}$ can be interpreted as the force applied by the noise [23-26]. According to theory, MPEPs are simply optimal trajectories that extend to the saddle point.

In Fig. 1, the theoretically predicted MPEP of (2) in the first quadrant, with $\mu=0.66$ and $\alpha=10$ (dotted curve), is compared with a typical escape path measured in the analog electronic model of (2) (jagged line). Three important, closely related, features deserve comment: (i) for $\mu<1$ the MPEP approaches the saddle point $(0,0)$ along the $y$ axis "ridge"; (ii) the shape of the MPEP in a boundary layer near the $y$ axis, obtained by linearizing the Hamiltonian dynamics at $(0,0)$, is close to $y \propto x^{\mu}$; (iii) the measured escape path departs from the MPEP and crosses the $y$ axis before reaching $(0,0)$.

Because of small transverse fluctuations, the MPEP may be viewed as being surrounded by a "WKB tube" with an asymptotically $(\epsilon \rightarrow 0)$ Gaussian profile of width $O\left(\epsilon^{1 / 2}\right)$ (see, e.g., [13,23]). In a sense the WKB tube is formed from optimal trajectories that are perturbations of the MPEP, which are more or less followed during actual escape attempts. Because the MPEP grazes the $y$ axis as it approaches $(0,0)$, the escaping particle should with overwhelming probability diffuse across $\partial \Omega$ before it reaches the saddle point, as observed (Fig. 1). One expects that the limiting exit location density will include a factor $\exp \left(-\right.$ const $\left.\times y^{2 / \mu} / \epsilon\right)$, arising from the Gaussian profile of the WKB tube. The exponent here is proportional to the square of the distance between the point $(0, y)$ and the closest point on the MPEP. One also expects the density to fall to zero as $y \rightarrow 0^{+}$.

The turning point approximation to the quasistationary density $\rho_{1}(x, y)$ that is valid near the saddle point $(0,0)$ turns out to be consistent with the expected behaviors. As noted, this approximation must match the WKB form that 
is valid elsewhere. It must also satisfy the approximate Fokker-Planck equation $\mathcal{L}_{\epsilon} \rho_{1} \approx 0$, with an absorbing boundary condition on the $y$ axis. It should be symmetric in $y$, since there are two incoming WKB tubes, just as there are two incoming MPEPs: one from the first quadrant and one from the fourth.

When $\mu<1$, the appropriate approximation to $\rho_{1}(x, y)$ near $(0,0)$ is necessarily [15]

$$
\begin{aligned}
\rho_{1}(x, y) \propto & |y|^{(1 / \mu)-1} \sinh \left(2 x|y / A|^{1 / \mu} / \epsilon\right) \\
& \times \exp \left(-|y / A|^{2 / \mu} / \epsilon\right),
\end{aligned}
$$

and it yields an exit location distribution of the abovementioned symmetrized Weibull form,

$$
p_{\epsilon}(y) d y \propto|y|^{(2 / \mu)-1} \exp \left(-|y / A|^{2 / \mu} / \epsilon\right) d y .
$$

Here the constant of proportionality is chosen to ensure unit total probability, and $A$ is the constant that appears in the behavior of the MPEPs near the saddle point (i.e., $\left.y= \pm A x^{\mu}\right)$. This distribution is localized on the $O\left(\epsilon^{\mu / 2}\right)$ length scale and contains the expected Gaussian factor. It is also bimodal.

Clear experimental evidence for the predicted splitting of the exit location distribution can be seen in the analog data of Fig. 2. It is evident that, for $\mu>1$ [Fig. 2(a)] escape occurs on average through the saddle point $(0,0)$ whereas, for $\mu<1$ [Figs. 2(b) and 2(c)] it does not: the bimodality of the exit location distribution is well demonstrated. Significant rounding of the minimum of $p_{\epsilon}$ at $y=0$ where the theory predicts $p_{\epsilon}=0$ is evident, however, in Fig. 2(b). This can be attributed to the noise intensities used in the experiment: the chain of reasoning leading to (5) is valid only in the $\epsilon \rightarrow 0$ limit. The rounding becomes less pronounced as $\mu$ and the angle of approach to the saddle decrease. The $\mu=0.2$ curve of Fig. 2(c) already resembles a symmetrized Weibull distribution.
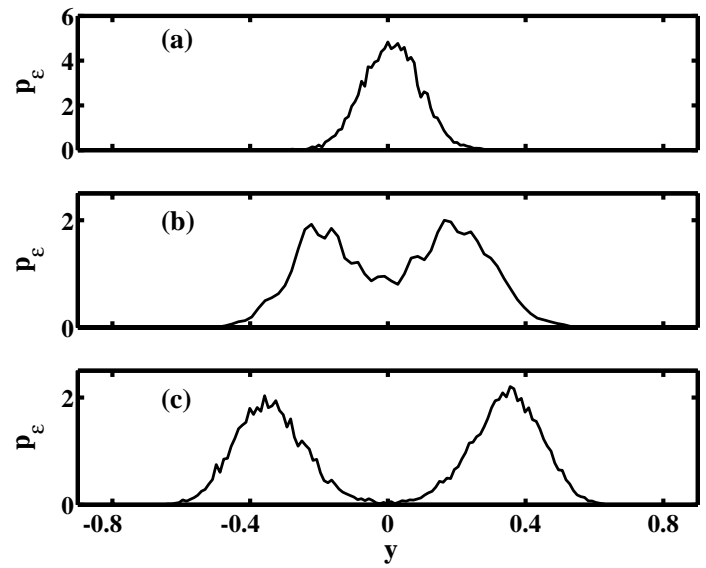

FIG. 2. Exit location distributions in analog simulation for $\alpha=10$ with (a) $\mu=2.0, \epsilon=0.034$; (b) $\mu=0.67, \epsilon=$ 0.011 ; and (c) $\mu=0.20, \epsilon=0.009$.
To facilitate quantitative comparison with the theory, measurements were made of partial components of the exit location distribution. Individual escape trajectories from the $x>0$ half-plane (Fig. 1) were selected according to their quadrants of origin and separately ensemble-averaged to build partial exit location distributions (whose sum obviously corresponds to the standard exit location distribution). Each partial component should display onesidedness, i.e., saddle-point avoidance, described by

$$
p_{\epsilon}(y) \propto \begin{cases}|y|^{(2 / \mu)-1} \exp \left(-|y / A|^{2 / \mu} / \epsilon\right), & y \geq 0 ; \\ 0, & y<0,\end{cases}
$$

i.e., an unsymmetrized, one-sided Weibull density.

Partial exit location distributions obtained by digital simulation for small $\epsilon$ are compared directly with the Weibull density (6) in Fig. 3. Agreement between theory and experiment is reasonable on the $O\left(\epsilon^{\mu / 2}\right)$ length scale and improves as $\epsilon$ decreases. On a smaller length scale near $(0,0)$, some "rounding" is evident as in Fig. 2, but it decreases as $\epsilon$ decreases.

There is a simple physical explanation of the limiting Weibull distribution, not dependent on the method of matched asymptotic approximations, which can be refined to yield a prediction for the length scale on which rounding occurs. Since

$$
\begin{aligned}
& \dot{x} \approx x+f_{x}(t), \\
& \dot{y} \approx-\mu y+f_{y}(t)
\end{aligned}
$$

near $(0,0)$, the $x$ and $y$ motions decouple. So exit through the $y$ axis is (in a sense) a one-dimensional activation phenomenon. Moreover, the figures show that $y$ is typically much larger than $x$ during an escape event, so to a first approximation $f_{y}$ may be disregarded. This implies that
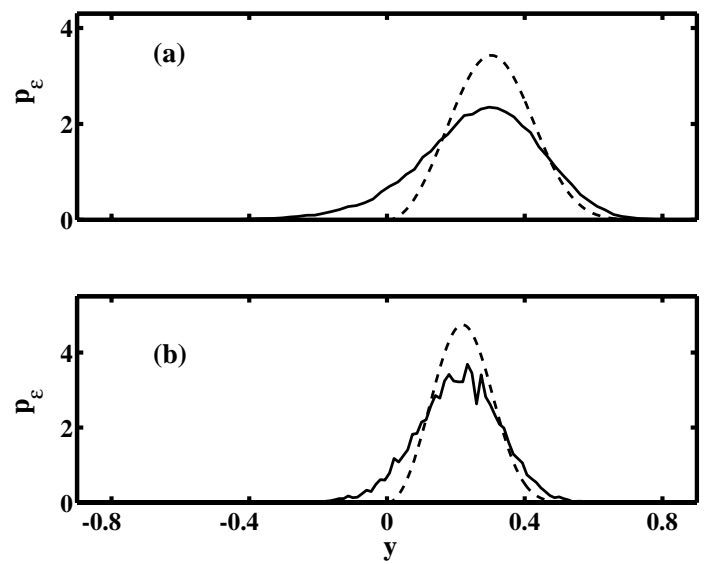

FIG. 3. Partial exit location distributions in digital simulation (full curves) compared with the theoretically predicted Weibull distributions (6) (dashed curves) for $\alpha=10$ and $\mu=0.66$, with (a) $\epsilon=0.04$ and (b) $\epsilon=0.015$. All curves, being graphs of probability density functions, are normalized to total area unity. The solid and dashed curves approach each other as $\epsilon \rightarrow 0$. 
$y(t) \approx y_{0} e^{-\mu t}$ for some $y_{0}$, and that the exit location distribution (i.e., the randomness in $y$ at exit time) is simply due to the variability of the exit time.

In any one-dimensional escape process activated by white noise, precise results are available on the weaknoise limit of the escape time, considered as a random variable $[13-15,27]$. The expected time to escape grows exponentially as $\epsilon \rightarrow 0$, but the duration of the final, successful escape attempt grows only logarithmically. If $\dot{x}=x$ near $x=0$ when $\epsilon=0$, the time for the particle to reach $x=0$ in its final escape attempt will grow in the $\epsilon \rightarrow 0$ limit as $\frac{1}{2} \log (1 / \epsilon)+\tau$, with

$$
P(\tau \leq t)=\exp \left(-e^{-2 t}\right)
$$

defining the cumulative distribution function of the comparatively small random quantity $\tau$.

Together, $y=y_{0} e^{-\mu t}$ and (9) imply that the exit location density on the $y$ axis must be of the Weibull form (6), with $A \equiv y_{0}$. A slight refinement of $y=y_{0} e^{-\mu t}$ yields a prediction for the rounding. Taking the force term $f_{y}(t)$ into account turns $y=y(t)$ into an Ornstein-Uhlenbeck process. The deterministic quantity $y_{0} e^{-\mu t}$ must be replaced by a Gaussian random variable of mean $y_{0} e^{-\mu t}$ and $O\left(\epsilon^{1 / 2}\right)$ standard deviation, thus smearing out the Weibull distribution on the $O\left(\epsilon^{1 / 2}\right)$ length scale, in qualitative agreement with the rounding in Figs. 2 and 3. In future experimental work, it may be possible to make the comparison quantitative.

The research of R.S. M. and D.L.S. was supported in part by NSF Grants No. DMS-9500792 and No. PHY9800979; that of D. G. L. and P.V.E. McC. in part by the U.K. Engineering and Physical Sciences Research Council (Grants No. GR/L01978, No. GR/L38875, and No. GR/L99562), INTAS (Project No. INTAS-96-0305), and the Royal Society of London; and that of R.M. and P.V.E. McC. in part by the Commission of the European Community Directorate General XII (Contract No. ERBCHBGCT940738).

[1] Noise in Nonlinear Dynamical Systems, edited by F. Moss and P.V.E. McClintock (Cambridge University Press, Cambridge, England, 1989), Vol. 1.

[2] M. Marder, Phys. Rev. E 54, 3442 (1996).

[3] N. Agmon and R. Kosloff, J. Phys. Chem. 91, 1988 (1987).
[4] M. M. Kłosek-Dygas, B. M. Hoffman, B. J. Matkowsky, A. Nitzan, M. A. Ratner, and Z. Schuss, J. Chem. Phys. 90, 1141 (1989).

[5] A. M. Berezhkovskii, V.Y. Zitserman, S.-Y. Shew, D.-Y. Yang, J. Kuo, and S.-H. Lin, J. Chem. Phys. 107, 10539 (1997).

[6] E. Stone and D. Armbruster, Noise and O(1) Amplitude Effects on Heteroclinic Cycles, Utah State University Report No. 980289 (to be published).

[7] D. Ludwig, SIAM Rev. 17, 605 (1975).

[8] M. Mangel, Theor. Population Biol. 45, 16 (1994).

[9] B.-Z. Bobrovsky and Z. Schuss, SIAM J. Appl. Math. 42, 174 (1982).

[10] A. L. Welti and B.-Z. Bobrovsky, IEEE Trans. Sel. Areas Comm. 8, 809 (1990).

[11] R. Landauer, Physica (Amsterdam) 194A, 551 (1993).

[12] R. Graham, in Ref. [1], Chap. 7.

[13] R. S. Maier and D. L. Stein, Phys. Rev. E 48, 931 (1993).

[14] M. V. Day, in Diffusion Processes and Related Problems in Analysis, edited by M. Pinsky (Birkhäuser, Boston/ Basel, 1990), Vol. 1, pp. 55-71.

[15] R. S. Maier and D. L. Stein, SIAM J. Appl. Math. 57, 752 (1997).

[16] R. S. Maier and D. L. Stein, Phys. Rev. Lett. 71, 1783 (1993).

[17] B.-Z. Bobrovsky and O. Zeitouni, Stoch. Process. Appl. 41, 241 (1992).

[18] M. V. Day, Stochastics 48, 227 (1994).

[19] R. S. Maier and D. L. Stein, J. Stat. Phys. 83, 291 (1996).

[20] D. G. Luchinsky, P. V.E. McClintock, and M. I. Dykman, Rep. Prog. Phys. 61, 889 (1998).

[21] R. Mannella, in Supercomputation in Nonlinear and Disordered Systems, edited by L. Vàzquez, F. Tirando, and I. Martin (World Scientific, Singapore, 1997), pp. $100-130$.

[22] T. Naeh, M. M. Kłosek, B. J. Matkowsky, and Z. Schuss, SIAM J. Appl. Math. 50, 595 (1990).

[23] M. I. Dykman, D. G. Luchinsky, P. V.E. McClintock, and V. N. Smelyanskiy, Phys. Rev. Lett. 77, 5229 (1996).

[24] D. G. Luchinsky and P. V.E. McClintock, Nature (London) 389, 463 (1997).

[25] D. G. Luchinsky, J. Phys. A 30, L577 (1997).

[26] D. G. Luchinsky, R. S. Maier, R. Mannella, P. V.E. McClintock, and D.L. Stein, Phys. Rev. Lett. 79, 3109 (1997).

[27] D. Ludwig, in Nonlinear Phenomena in Physics and Biology, edited by R.H. Enns, B.L. Jones, R. M. Miura, and S. S. Rangnekar (Plenum, New York, 1981), pp. 549-566. 